\title{
"Histopathological Pattern of Chronic Arterial Occlusive Disease among the Adult Population Attending a Tertiary Medical Facility in Bangladesh (BSMMU), Dhaka, Bangladesh"
}

\author{
A. K. Al Miraj ${ }^{1 *}$, H. N. Ashikur Rahaman², Md. Magfur Rahman ${ }^{3}$, Md. Saif Ullah Khan ${ }^{4}$, Mr. Md. Anwarul Islam
}

\author{
${ }^{1}$ Research Assistant, Department of Vascular Surgery, Bangabandu Sheikh Mujib Medical University (BSMMU), Dhaka, Bangladesh \\ ${ }^{2}$ Registrar, Dept. of Clinical Oncology, Enam Medical College \& Hospital, Savar, Dhaka, Bangladesh \\ ${ }^{3}$ Cardiac Surgeon \& Consultant Cardiologist \& Diabetologist, Department of Cardiac Surgery, Bangabandu Sheikh Mujib Medical University \\ (BSMMU), Dhaka, Bangladesh \\ ${ }^{4}$ Associate Professor \& Chairman, Department of Vascular Surgery, Bangabandu Sheikh Mujib Medical University (BSMMU), Dhaka, Bangladesh \\ ${ }^{5}$ Research Assistant, Department of Cardiac Surgery, Bangabandu Sheikh Mujib Medical University (BSMMU), Dhaka, Bangladesh
}

Introduction: Atherosclerotic coronary artery disease (CAD) is of great concern in young adults because of its potential to cause great incapacitation. This arena of cardiology has gained importance in South Asian countries, particularly Bangladesh due to increased prevalence that is related to traditional risk factors, altered life styles and inherent risk factors. Objective: To see the histopathological pattern of chronic arterial occlusive disease such atherosclerotic, thrombotic, vacuities. Materials and methods: The patients having surgery in Department of Vascular Surgery in BSMMU during from July 2017 to Jun 2018. A full clinical history along with routine and special investigations Histopathological examination report. This study will help to observed pattern of chronic arterial occlusive disease adult age group. The investigation's and treatment facilities presently available in Bangabandhu Sheikh Mujib Medical University will be utilized. Additional facility in required for histopathlogical examination, which will be done from other reputed pathological laboratory also. A 1-year retrospective autopsy-based study was carried out in a BSMMU and patients aged<45 years with IHD were selected. Results: Out of 201 autopsied cases of IHD, 95 patients (17.4\%) were young. Among these 95 patients, 84 (88.4\%) had IHD related to atherosclerotic CAD; the youngest patient was 18 years old. Predictably there was sole involvement of left anterior descending artery and the presence of fibrous plaques. Irrespective of the plaque morphology, the commonest complication was thrombosis produced by plaque erosion seen in $36.9 \%$ of patients. Acute coronary insufficiency was noted in 52 patients $(61.9 \%)$, while healed infarctions were surprisingly noted in 28 patients $(33.3 \%)$. Screening for IHD in the young population may help to improve prognosis by detecting subclinical disease, although more studies are necessary to establish reference limits for this young population. Additional research must also focus on treatment concerns that are specific to young patients. Conclusion: This study reiterates the necessity of a definitive protocol to be setup for detailed postmortem examination since significant atherosclerosis can lead to sudden deaths even in the young population. Such a meticulous postmortem coupled with histopathological examination minimizes the risk of autopsy being negative or obscure one.

Keywords: Atherosclerosis; Vasculitis; Thromboangitis obliterates; Aging.

Copyright @ 2020: This is an open-access article distributed under the terms of the Creative Commons Attribution license which permits unrestricted use, distribution, and reproduction in any medium for non-commercial use (NonCommercial, or CC-BY-NC) provided the original author and source are credited.

\section{INTRODUCTION}

The management of the patient with chronic arterial occlusive disease (CAOD) has to be planned in the context of the epidemiology of the disease, its natural history and, in particular, the modifiable risk factors for the systemic disease as well as those that predict deterioration of the circulation to the limb. Atherosclerosis disease is a major health problem in the elderly population in the world to date; most research has focused on the coronary and cerebral manifestations of the disease. Chronic arterial occlusive disease (CAOD) has received less attention. Noncommunicable diseases are disorders of long duration and slow progression. Important among them are cardiovascular diseases (CVD), cancers, chronic respiratory diseases and diabetes mellitus (DM). Collectively, besides producing morbidity and 
disability, they are the leading causes of global mortality. A similar scenario exists in India, where CVDs account for nearly $30 \%$ of such disorders [1]. The underlying pathology in most patients in the Indian sub-continent is atherosclerosis, whose progression and/or acceleration is proportional to the traditional risk factors, altered life styles as well as inherent risk factors [1]. Obliterative endarteries and Atherosclerosis disease is a major health problem in the elderly population in the world to date; most research has focused on the coronary and cerebral manifestations of the disease. Lower extremity arterial disease (LEAD) has received less attention. Populations most affected are from lowand middle-income countries like Bangladesh, where $80 \%$ of these deaths occur [21]. However, the use of simple objective non-invasive diagnostic techniques in recent population-based studies has allowed identification of asymptomatic LEAD and shown that the prevalence of disease is several fold higher than previously estimated. The prevalence increases sharply with age, from $3 \%$ in those fewer than 60 years to over $20 \%$ at $75+$ years in U.S. Detection of LEAD in the elderly provides a rapid, easy assessment of the level of atherosclerosis in the body and identifies those at high risk for mortality and morbidity. LEAD is associated with a relative risk of 4 to 5 for all-cause mortality. In about $25 \%$ of those with LEAD, the disease progresses over time, leading to loss of mobility, gangrene or amputation. In this study will try to see the pattern of LEAD in all adult group patients operated in department of vascular surgery of BSMMU. This review summarizes current knowledge regarding the etiology and natural history of LEAD from an epidemiologic viewpoint, delineating areas in which additional research is needed. By and large, this atherosclerotic process culminates in ischemic heart disease (IHD) and a worrisome fact is an increasing incidence of IHD in the young population $(\leq 45$ years of age) [2]. Furthermore, the initial manifestation of IHD in these young persons may be in the form of sudden cardiac death (SCD). In this study, we sought to evaluate, at autopsy, the pathology of atherosclerotic coronary artery disease (CAD) in young patients with IHD. A complete autopsy examination was carried out in all the cases according to standard uniform guidelines practicing in our country. SCD was defined as an unexpected natural death occurring within $1 \mathrm{~h}$ after onset of symptoms in an apparently healthy subject or in one whose disease was not so severe as to predict an abrupt outcome [3]. When un-witnessed, it referred to death of an individual within $24 \mathrm{~h}$ after being seen alive and in a normal state of health. It also included patients with sudden rapid deterioration, who would have an illness, not always expected to cause death.

\section{OBJECTIVE}

To see the histopathological pattern of chronic arterial occlusive disease such atherosclerotic, thrombotic, vacuities

\section{MATERIALS AND METHODS}

The patients having surgery in Department of Vascular Surgery in BSMMU during from July 2017 to Jun 2018. A full clinical history along with routine and special investigations Histopathological examination report. This study will help to observed pattern of chronic arterial occlusive disease adult age group. The investigation's and treatment facilities presently available in Bangabandhu Sheikh Mujib Medical University will be utilized. Additional facility in required for histopathlogical examination, which will be done from other reputed pathological laboratory also. In a 1year retrospective autopsy-based study in a BSMMU (catering to largely the lower socio-economic group), all cases of IHD that caused death or was identified as a co-morbid condition were reviewed and patients aged 45 years were selected for further analyses. The degree of stenosis was classified into three categories: less than $50 \%$; equal to or more than $50 \%$, but less than $75 \%$, and equal to or greater than $75 \%$ (critical stenosis). The fibrous plaques showed smooth muscle proliferation in a background of collagen and proteoglycans, while the fatty plaques were composed a large lipid-rich core separated from the lumen by a thin fibrous cap. The lipid component was formed of collections of foamy macrophages and/or extra-cellular lipid material. The fibro fatty plaques consisted of an equal proportion of fibro-cellular and lipid elements. Presence of complications and other histological changes such as calcification and inflammation were also noted. Depending on the morphology of the myocardium, IHD was classified as acute coronary insufficiency (ACI, critical coronary stenosis devoid of visible ischaemic changes), acute myocardial infarction (AMI), acute-onchronic myocardial infarction (ACMI) and chronic myocardial infarction (CMI). These were correlated with the clinical features, risk factors and investigations (obtained from the inquest papers and/or hospital records) in two age groups 30 years and from 31-45 years. All data analysis Windows SPSS Version 16.0.

\section{RESULTS}

This study was 201 autopsied cases of IHD; 95 patients $(17.4 \%$ ) were young (45 years). In 11 of these patients, the IHD had been related to abnormal coronary ostial localizations, coronary vasculitis, vasospasm, left ventricular hypertrophy and hypercoagulability. The remaining 84 patients $(15.4 \%)$ had atherosclerotic CAD and were further analysed; only 13 were women $(15.5 \%)$. The youngest patient was 18 years old. Patient's $\leq 30$ years. There were 14 patients in this group (16.7\%), including 12 males and 2 females with an age range of 18 to 30 years (mean age of 26.8 years); only three patients had hospital admissions for a period of 2-5 days. Dyspnoea and/or chest pain were the presenting symptoms in nine patients. Two patients apparently "dropped dead", one of whom was undergoing a pleural tapping. Two others developed sudden-onset unconsciousness, with preceding generalized tonic clonic convulsions in one of them. 
Another patient, an 18-year-old girl with systemic lupus erythematosus (SLE), had been admitted for acute pancreatitis. Hypertension had been present in two other patients. The other risk factors and autopsy findings (if any) are enlisted in [Table 1].

Table-1: Risk factors and autopsy findings in patients $\leq 30$ years $(\mathrm{N}=14)$

\begin{tabular}{|l|l|l|}
\hline Risk factors & Autopsy findings- cardiac & Autopsy findings - non-cardiac \\
\hline Hypertension $(\mathrm{n}=2)$ & ----------------------------------- & Respiratory bronchiolitis $(\mathrm{n}=1)$ \\
Diabetes mellitus $(\mathrm{n}=1)$ & --------- & Pulmonary tuberculosis $(\mathrm{n}=1)$ \\
Systemic lupus erythematosus $(\mathrm{n}=1)$ & & $\begin{array}{l}\text { Lupus pneumonitis }(\mathrm{n}=1) \\
\text { Alcoholic steatosis }(\mathrm{n}=1)\end{array}$ \\
& & $\begin{array}{l}\text { Nodular regenerative hyperplasia }(\mathrm{n}=1) \\
\text { Acute pancreatitis }(\mathrm{n}=1)\end{array}$ \\
\hline
\end{tabular}

Table-2: Pattern of IHD and coronary atherosclerosis in patients $\leq 30$ years $(\mathrm{N}=14)$

\begin{tabular}{|l|l|l|l|}
\hline & Type of plaque & Fatty \\
\hline Type of IHD & Fibrous & Fibro-fatty & Erosion with fresh \\
ACI $(\mathrm{n}=12)$ & Uncomplicated $(\mathrm{n}=1)$ & $\begin{array}{l}\text { Uncomplicated }(\mathrm{n}=3) \\
\text { Erosion with fresh thrombus }(\mathrm{n}=4)\end{array}$ & $\begin{array}{l}\text { Erombus }(\mathrm{n}=2) \\
\text { throm }\end{array}$ \\
\hline CMI $(\mathrm{n}=2)$ & Erosion with fresh thrombus $(\mathrm{n}=2)$ & Uncomplicated $(\mathrm{n}=1)$ & \\
\hline
\end{tabular}

The heart weights ranged from 220 to $350 \mathrm{~g}$ (mean weight of $277.9 \mathrm{~g}$ ). In all these patients, the left anterior descending artery (LAD) was involved by atherosclerosis (mean length of $1.3 \mathrm{~cm}$ ). Majority of the patients (12 patients) had ACI (Table 2). This was related to critical atherosclerotic stenosis in four patients, while erosion with occlusive thrombus was seen in eight patients. They occurred in plaques, which had produced critical (75\% in five arteries) or noncritical stenosis $(50 \%$ in three arteries). The healed infarction in two patients showed fibro-fatty plaque with critical stenosis and an eroded fibrous plaque with organized thrombus, respectively.

Table-3: Risk factors and autopsy findings in patients 31 to 45 years $(\mathrm{N}=70)$

\begin{tabular}{|c|c|c|}
\hline Risk factors & Autopsy findings - cardiac & Autopsy findings - non-cardiac \\
\hline $\begin{array}{l}\text { Hypertension }(\mathrm{n}=22) \\
\text { Diabetes mellitus }(\mathrm{n}=15) \\
\text { Chronic alcoholism }(\mathrm{n}=11) \\
\text { Smoking }(\mathrm{n}=5) \\
\text { Obesity }(\mathrm{n}=1) \\
\text { Systemic lupus } \\
\text { erythematosus }(\mathrm{n}=1)\end{array}$ & $\begin{array}{l}\text { Patent foramen ovale }(n=1) \\
\text { Secundum atrial septal defect }(n=1) \\
\text { Rheumatic heart disease }(n=1) \\
\text { Arrhythmogenic cardiomyopathy } \\
(n=1)\end{array}$ & $\begin{array}{l}\text { Meningioma }(\mathrm{n}=1) \text {; Pulmonary hemorrhage } \\
(\mathrm{n}=1) \text { Respiratory bronchiolitis }(\mathrm{n}=1) \text {; } \\
\text { Pulmonary tuberculosis }(\mathrm{n}=1) \text {; Pulmonary } \\
\text { sarcoidosis }(\mathrm{n}=3) \text {; Invasive aspergillosis }(\mathrm{n}=1) \text {; } \\
\text { Lupus pneumonitis }(\mathrm{n}=1) \text {; Bronchiectasis } \\
(\mathrm{n}=1) \text {; Primary pulmonary hypertension }(\mathrm{n}=1) \text {; } \\
\text { Intestinal gangrene }(\mathrm{n}=6) \text {; Alcoholic steatosis } \\
(\mathrm{n}=6) ; \text { Alcoholic cirrhosis } \quad(\mathrm{n}=2) ; \quad \text { Acute } \\
\text { pancreatitis } \\
(\mathrm{n}=5) ; \text { Acute tubular necrosis }(\mathrm{n}=1) ; \\
\text { Renal infarction }(\mathrm{n}=1) \text {; Acute pyelonephritis } \\
(\mathrm{n}=2) ; \text { Crescentic glomerulonephritis }(\mathrm{n}=1) ; \\
\text { Lupus nephritis }(\mathrm{n}=1) \text {; Focal segmental } \\
\text { glomerulosclerosis }(\mathrm{n}=2) \text {; Diabetic nephropathy } \\
(\mathrm{n}=4) ; \text { Benign nephrosclerosis }(\mathrm{n}=3) \text {; Splenic } \\
\text { infarction }(\mathrm{n}=1)\end{array}$ \\
\hline
\end{tabular}

Among 70 patients in this group, there were only 11 women $(15.7 \%)$; mean age was 39.5 years. Dyspnoea with or without chest pain were the presenting symptoms in 33 patients. Ten patients were brought in an unconscious state, while three others collapsed during exertional activities. A 34-year-old woman developed cardiac arrest after administration of general anaesthesia for obstructed labour. Vomiting had been the only symptom in three patients. The remaining 20 patients had been admitted for acute abdomen, acute febrile illness, peripheral vascular disease and limb cellulitis, followed by sudden clinical deterioration. Hypertension and/or diabetes mellitus were present in 32 patients with a past history of IHD in four and cerebrovascular accident in one. The other risk factors and autopsy findings (if any) are enlisted in [Table 3]. 


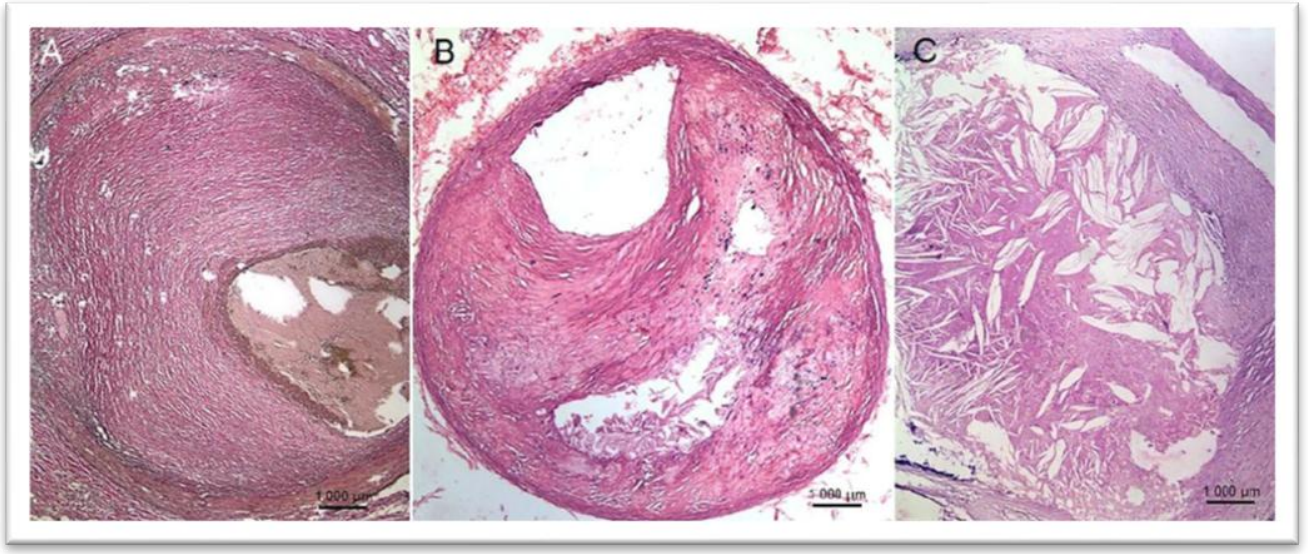

Fig-1A, B, C

Figure 1: (A) Fibrous plaque, which is fibrocellular and also rich in proteoglycans (Elastic van Gieson, 100); (B) Fibro-fatty plaque, composed of an equal proportion of fibro-cellular and lipid elements (HE, 100); (C) Fatty plaque, comprising a large lipidrich core separated from the lumen by a thin fibrous cap. The lipid material may in the form of collections of foamy mac-rophages and/or extra-cellular lipid material (HE, 100).
The major epicardial arteries had been studied by serial crosssections at an interval of $0.5 \mathrm{~cm}$ and the sites of maximal narrowing or occlusion were taken for histopathology with inclusion of arterial segments, proximal and distal to stenosis/occlusion as well. The plaques were simplistically categorized as fibrous, fibro-fatty and fatty [Figure 1].

Table-4: Pattern of IHD and coronary atherosclerosis in patients 31 to 45 years $(N=70)$

\begin{tabular}{|c|c|c|c|}
\hline & \multicolumn{3}{|l|}{ Type of plaque } \\
\hline Type of IHD & Fibrous & Fibro-fatty & Fatty \\
\hline $\mathrm{ACI}(\mathrm{n}=40)$ & $\begin{array}{l}\text { Uncomplicated }(n=12) \text { (anomalous } \\
\text { origin of left anterior descending artery } \\
\text { from right coronary }(n=1)) \\
\text { Erosion with fresh thrombus }(n=4) \\
\text { Erosion with organized thrombus }(n=4) \\
\text { Rupture with fresh thrombus }(n=1)\end{array}$ & $\begin{array}{l}\text { Uncomplicated }(\mathrm{n}=6) \\
\text { Intra-plaque haemorrhage } \\
(\mathrm{n}=1) \\
\text { Erosion with fresh } \\
\text { thrombus }(\mathrm{n}=6) \\
\text { Erosion with recanalized } \\
\text { thrombus }(\mathrm{n}=1)\end{array}$ & $\begin{array}{l}\text { Uncomplicated }(n=3) \\
\text { Erosion with fresh } \\
\text { thrombus }(n=1) \\
\text { Rupture with fresh } \\
\text { thrombus }(n=1)\end{array}$ \\
\hline AMI $(n=4)$ & Uncomplicated $(\mathrm{n}=1)$ & $\begin{array}{l}\text { Uncomplicated }(\mathrm{n}=1) \\
\text { Erosion with fresh } \\
\text { thrombus }(\mathrm{n}=1)\end{array}$ & $\begin{array}{l}\text { Uncomplicated }(n=1) \\
\text { Erosion with fresh } \\
\text { thrombus }(n=1)\end{array}$ \\
\hline A-CMI $(n=5)$ & $\begin{array}{l}\text { Uncomplicated }(\mathrm{n}=2) \\
\text { Erosion with organized thrombus }(\mathrm{n}=1)\end{array}$ & $\begin{array}{l}\text { Erosion with fresh } \\
\text { thrombus }(\mathrm{n}=2)\end{array}$ & \\
\hline CMI $(n=21)$ & $\begin{array}{l}\text { Uncomplicated }(\mathrm{n}=4) \\
\text { Erosion with fresh/organized thrombus } \\
(\mathrm{n}=1) \\
\text { Erosion with fresh/recanalized thrombus } \\
(\mathrm{n}=1) \\
\text { Erosion with organized thrombus }(\mathrm{n}=3)\end{array}$ & $\begin{array}{l}\text { Uncomplicated }(\mathrm{n}=4) \\
\text { Erosion with fresh } \\
\text { thrombus }(\mathrm{n}=2) \\
\text { Erosion with organized } \\
\text { thrombus }(\mathrm{n}=4)\end{array}$ & $\begin{array}{l}\text { Uncomplicated }(\mathrm{n}=1) \\
\text { Rupture with } \\
\text { fresh/organized } \\
\text { thrombus } \\
(\mathrm{n}=1)\end{array}$ \\
\hline
\end{tabular}

The heart weights ranged from 180 to $540 \mathrm{~g}$ (mean weight of $324.1 \mathrm{~g}$ ). Single arterial affection was present in 59 hearts $(84.3 \%)$ and involved the LAD artery in most cases (43 hearts, $72.9 \%$; mean length of $1.1 \mathrm{~cm}$ ); two or more epicardial arteries were affected in the remaining 16 cases $(11.4 \%)$. One patient also had an anomalous origin of the LAD from the right coronary. The plaques had been fibrous in $34(48.6 \%)$, fibrofatty in $28(40.0 \%)$ and fatty in eight patients $(11.4 \%)$ [Table 4$]$.
Critical atherosclerotic stenosis was present in the arteries in 56 hearts $(80.0 \%)$. ACI, observed in 40 patients, resulted from mere critical stenosis (21 arteries), from thrombotic occlusions related to erosions (16 plaques, Figure 2) or ruptures (two plaques) and intra-plaque haemorrhage (one fibrofatty plaque). AMI and A-CMI were seen in four and five patients, respectively. They were seen with critical stenosis (two arteries) and thrombotic occlusions (five 
arteries); in two patients, the plaques were found to have produced non-critical stenosis despite presence of infarctions. There were a good number of patients with healed infarctions, $21(30.0 \%)$, related to critical stenosis (nine arteries) and stenosis with thrombotic complications (12 arteries). Associated calcification in the atherosclerotic plaques was present mainly towards the basal aspects in eight arteries. There was inflammatory infiltrate in the plaque and the adjoining media in 24 arteries, while associated adventitial fibrosis and inflammation was present in 21 arteries. All these changes were prominently seen with fibro-fatty or fatty plaques.

\section{DISCUSSION}

Arterial vascular disease is usually due to age and traditional risk factors related to atherosclerosis. However, vasculitis and other uncommon no inflammatory arteriopathies can present with both ischemic symptoms from occlusive disease and degenerative lesions such as aneurysms, requiring specific diagnostic evaluation and treatment. Population-based surveillance system to track noncommunicable chronic disease is currently absent in Bangladesh. In addition, it is hard to find accurate information on the prevalence of diseases in Bangladesh due to lack of national population-based surveys or central administrative health data. A limited number of studies have been undertaken on the prevalence of CVD in the Bangladeshi population of which only two discussed the burden of CVD [22-27]. The disorders reviewed in this chapter can be distinguished from atherosclerotic disease by several clinical features, including young age at presentation and paucity of traditional risk factors. In some cases, another clue to vasculitis is a systemic constitutional syndrome of fevers, chills, night sweats, or unexplained weight loss. Evidence of a multisystem disorder on examination or laboratory testing, with elevated inflammatory biomarkers, is also suggestive. A temporal association with a new drug may suggest drug-related vasospasm or vasculitis. A positive family history is a useful clue to an inherited vasculopathy such as pseudoxanthomal elasticum or neurofibromatosis. In young adults in general aged 18 to 45 years [4], CVD manifest as both congenital and acquired dis- orders [5]. The cardiac abnormalities can result in SCD, a devastating event for the family as well as from the societal perspective. We reported a cohort of 84 young individuals who showed features of IHD at autopsy due to coronary arterial atherosclerosis. These cases accounted for about $15.4 \%$ of all autopsied cases of IHD, causing SCD; $16.7 \%$ were below the age of 30 years. However, this may not represent the true incidence as many of them had not been submitted for a detailed pathological examination, which is extremely essential in young SCDs [6]. The process of atherosclerosis has been known since times immemorial [7] and it has come a long way from being called a "degenerative and proliferative" intimal disease to a chronic inflammatory response to multi-factorial endothelial injury [8]. In general, there seems to be a global rise in the incidence and prevalence of atherosclerotic diseases. But, a premature and rapidly progressive ("malignant") involvement of the coronary arteries occurs in South Asia, particularly India (15.4\% in our series), and this is seen not only in individuals settled in that region but also in the immigrant population, with a lower incidence of less than $3 \%$ seen in the Western population [9]. Predictably, males $(84.5 \%)$ outnumbered women $(15.5 \%)$. Hypertension, DM, smoking, dyslipidemia, obesity, sedentary life style and positive family history continue to be the traditional risk factors [4, 9]. Hypertension and/or DM was noted in $40.5 \%$, while a history of smoking was documented in only five patients. These were often present in patients over 30 years of age. Interestingly, hyper- tension was made as a post-mortem diagnosis in five patients on the basis of concentric left ventricular hypertrophy and accompanying early nephrosclerosis changes, indicating a sub-clinical condition. The other non-traditional factors include polymorphisms of genes coding for molecules involved in the lipid metabolism, altered diet- ary habits, acute phase reactants, micro-organisms, endocrinal disorders, collagen vascular disorders and excessive alcoholic intake $[4,9,10]$. Substances of abuse also predispose to IHD in young patients, due to not only vasopasm but also premature atherosclerosis and its complications [11], but we did not have any such case in this cohort of patients. Among the 13 women in this study, five of them were pregnant with presence of pregnancy induced hypertension in four. Pregnancy is increasingly being recognized as a cause of cardiovascular and metabolic stress [12]. It is also important to remember the psycho-biological aspects, which do not necessarily fall into the purview of the health- care systems [13]. These social determinants can lead to stress, which fosters unhealthy habits and neuroendocrine perturbations with alterations in plaque morphology [14]. Inflammatory cells have always been part and parcel of atherosclerosis. Through release of cytokines, they produce modification, progression and subsequent complications, inducing a constellation of symptoms in the patient. Much prominence has been given to the accumulations of lipids. However, such fatty plaques were noted in only 10 of the arterial segments $(12 \%)$ studied, which reiterates the fact that lipid-rich plaques are seen in older patients [15]. In the remaining $88 \%$, the plaques were either fibrous or fibro-fatty with little calcification. In concordance with literature [16-18], single artery involvement (73 cases, $86.9 \%$ ) and involvement of the left anterior descending artery (57 cases, 67\%) was also noted in our series. Thrombotic occlusion was seen in 43 arteries $(51.2 \%)$, and fibrous plaques were involved in 18 of these arteries (41.9\%). This is in contrast to the study by Corrado et al. [19], where only $27 \%$ of the arteries had thrombosis, particularly in non-fibrous plaques. Majority of the plaques (36.9\%) had 
thrombotic occlusion, related to plaque erosion, which does not necessarily rely on the traditional risk factors for its pathogenesis. The erosions are characterized by denudation or disruption of the endothelium that may be related to the haemodynamics of blood flow in the region of the plaque or through cytokines released by T- lymphocytes, macrophages and granulocytes; the latter appears to be aided by the proteoglycan-rich plaques, frequently seen in the young [20]. An interesting finding noted was the presence of healed infarcts in nearly one-third of the young patients (mostly above 30 years of age). IHD had been diagnosed in only four of these patients. This indicates that in the remaining patients, the symptoms could have been ignored or attributed to some other ailment, particularly acid-peptic disease.

\section{CONCLUSION}

This study reiterates the necessity of a definitive protocol to be setup for detailed postmortem examination since significant atherosclerosis can lead to sudden deaths even in the young population. Such a meticulous postmortem coupled with histo-pathological examination minimizes the risk of autopsy being negative or obscure one. The study can be of great help in convincing and counselling bereaved relatives of deceased about cause and manner of death, so that the subtle yet alarming signs and symptoms are not ignored. A strategy for screening and optimal medical therapy can also be worked out in the near future.

\section{REFERENCES}

1. Prabhakaran D, Jeemon P, Roy A. Cardiovascular diseases in India: current epidemiology and future directions. Circulation. 2016; 133:1605-1620.

2. Rao M, Xavier D, Devi O. Prevalence, treatments and outcomes of coronary artery diseases in Indians: a systematic review. Indian Heart J. 2015; 67:302-310.

3. Basso C, Aguilera B, Banner J. Guidelines for autopsy investigation of sudden cardiac death: 2017 update from the Association for European Cardiovascular Pathology. Virchows Arch. 2017; 471:691-705.

4. Aggarwal A, Srivastava S, Velmurugan M. Newer perspectives of coronary artery disease in young. World J Cardiol. 2016; 8:728-734.

5. Andersson C, Vasan RS. Epidemiology of cardiovascular disease in young individuals. Nat Rev Cardiol. 2018; 15:230-240.

6. De Noronha SV, Behr ER, Papadakis M. The importance of specialist cardiac histopathological examination in the investigation of young sudden cardiac deaths. Europace. 2014; 16:899-907.

7. Clarke EM, Thompson RC, Allam AH. Is atherosclerosis fundamental to human aging? Lessons from ancient mummies. J Cardiol. 2014; 68:329-334.

8. Falk E. Pathogenesis of atherosclerosis. J Am Coll Cardiol. 2006; 47:C7-C12.
9. Ahmed ST, Rehman H, Akeroyd JM. Premature coronary artery disease in South Asians: burden and determinants. Curr Atheroscler Rep. 2018; 20:6.

10. Shokeen D, Aeri BT. Risk factors associated with the increasing cardiovascular diseases prevalence in India: a review. J Nutr Food Sci. doi: 10.4172/ 2155-9600.1000331

11. Fischbach $P$. The role of illicit drug use in sudden death in the young. Cardiol Young. 2017; 27:S75S79.

12. Sahni G. Chest pain syndromes in pregnancy. Cardiol Clin. 2012; 30:343-367.

13. Havranek EP, Mujahid MS, Barr DA. Social determinants of risk and outcomes for cardiovascular disease: a scientific statement from the American Heart Association. Circulation. 2015; 132:873-898.

14. Yang Y, Bi M, Xiao L. Perceived stress sta- tus and sympathetic nervous system activation in young male patients with coronary artery disease in China. Eur J Intern Med. 2015; 26: 726730 .

15. Kitulwatte ID, Pollanen MS. A comparative study of coronary atherosclerosis in young and old. Am J Forensic Med Pathol. 2015; 36:323-326.

16. Rubin JB, Borden WB. Coronary heart disease in young adults. Curr Atheroscler Rep. 2012; 14: 140-149.

17. Alkhawam H, Zaiem F, Sogomonian R. Coronary artery disease in young adults. Am J Med Sci. 2015; 350:479-483.

18. Green AC, Sheppard MN. Sudden cardiac death associated with premature atheroma in the young: an autopsy study emphasizing single-vessel lesions. Cardiol Young. 2016; 26:743-748.

19. Corrado D, Basso C, Poletti A. Sudden death in the young. Is acute coronary thrombosis the major precipitating factor? Circulation. 1994; 90: 23152323.

20. Partida RA, Libby P, Crea F. Plaque erosion: A new in vivo diagnosis and a potential major shift in the management of patients with acute coronary syndromes. Eur Heart J. 2018; 39:2070-2076.

21. Laslett LJ, Alagona P, Clark BA. The worldwide environment of cardiovascular disease: prevalence, diagnosis, therapy, and policy issues: a report from the American college of cardiology. J Am Coll Cardiol. 2012;60(25):S1-S49.

22. Zaman MM, Ahmed J, Choudhury SR, Numan SM, Parvin K, Islam MS. Prevalence of ischemic heart disease in a rural population of Bangladesh. Indian Heart J. 2007;59(3):239-241.

23. Sayeed MA, Mahtab H, Sayeed S, Begum T, Khanam PA, Banu A. Prevalence and risk factors of coronary heart disease in a rural population of Bangladesh. Ibrahim Med Coll J. 2010;4(2):37-43.

24. Parr JD, Lindeboom W, Khanam MA, Koehlmoos TL. Diagnosis of chronic conditions with modifiable lifestyle risk factors in selected urban 
and rural areas of Bangladesh and sociodemographic variability therein. BMC Health Serv Res. 2011;11(1):309.

25. Van Minh H, Ng N, Juvekar S. Self-reported prevalence of chronic diseases and their relation to selected sociodemographic variables: a study in INDEPTH Asian sites, 2005. Prev Chronic Dis. 2008;5(3):A86
26. Turin TC, Shahana N, Wangchuk LZ. Burden of cardio-and cerebro-vascular diseases and the conventional risk factors in South Asian population. Glob Heart. 2013;8(2):121-130.

27. Al Mamun M, Rumana N, Pervin K. Emerging burden of cardiovascular diseases in Bangladesh. J Atheroscler Thromb. 2016;23(4):365-375. 